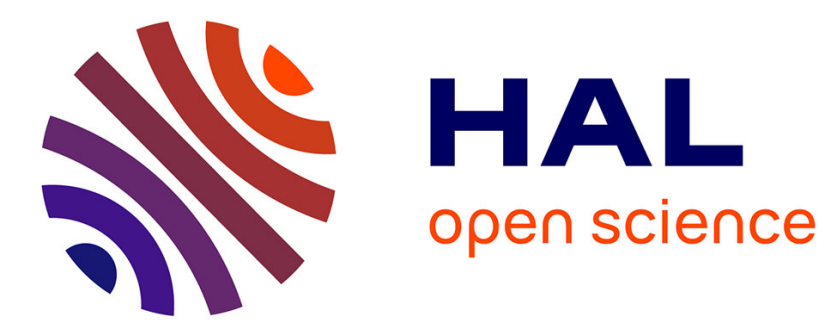

\title{
Investigation of low field response of metamagnetic Heusler alloys as multiphysic memory alloys
}

\author{
Mickaël Null Lallart, Hiroyuki Miki, Linjuan Yan, Gildas Diguet, Makoto
}

Ohtsuka

\section{- To cite this version:}

Mickaël Null Lallart, Hiroyuki Miki, Linjuan Yan, Gildas Diguet, Makoto Ohtsuka. Investigation of low field response of metamagnetic Heusler alloys as multiphysic memory alloys. Journal of Physics D: Applied Physics, 2020, 53 (34), pp.345002. 10.1088/1361-6463/ab8c7c . hal-02970976

\section{HAL Id: hal-02970976 https://hal.science/hal-02970976}

Submitted on 22 Oct 2020

HAL is a multi-disciplinary open access archive for the deposit and dissemination of scientific research documents, whether they are published or not. The documents may come from teaching and research institutions in France or abroad, or from public or private research centers.
L'archive ouverte pluridisciplinaire HAL, est destinée au dépôt et à la diffusion de documents scientifiques de niveau recherche, publiés ou non, émanant des établissements d'enseignement et de recherche français ou étrangers, des laboratoires publics ou privés. 


\title{
Investigation of Low Field Response of Metamagnetic Heusler Alloys as Multiphysic Memory Alloys
}

\author{
Mickaël LALLART ${ }^{1,2,3}$, Hiroyuki MIKI ${ }^{1}$, Linjuan YAN $^{1,3}$, \\ Gildas DIGUET ${ }^{3}$ and Makoto OHTSUKA ${ }^{4}$ \\ ${ }^{1}$ Institute of Fluid Science, Tohoku University, 2-1-1, Katahira, Aoba-ku, Sendai, \\ Miyagi 980-8577, Japan \\ ${ }^{2}$ Univ. Lyon, INSA-Lyon, LGEF EA 682, F-69621, France \\ ${ }^{3}$ ELyTMaX UMI 3757, CNRS - Université de Lyon - Tohoku \\ University,International Joint Unit, Tohoku University, Sendai, Japan \\ ${ }^{4}$ Institute of Multidisciplinary Research for Advanced Materials, Tohoku \\ University, 2-1-1, Katahira, Aoba-ku, Sendai 980-8577, Japan \\ E-mail: mickael.lallart@insa-lyon.fr
}

\begin{abstract}
Extending the concept of Shape Memory Alloys (SMAs), MultiPhysic Memory Alloys (MPMAs) based on Heusler compounds show remarkable properties in terms of multiphysic transitions coupling thermal, structural and magnetic domains. Such characteristics thus unveil new application potentials in the field of actuation or energy harvesting for instance. In particular, one of the most notable features of such materials lies in the presence of a magnetic response in a particular temperature range. In order to further investigate the origin of such characteristics, this study aims at providing qualitative and quantitative theoretical insights for shaping potential future material developments and tailoring, along with experimental investigations supporting the proposed theoretical framework. Such a development is done by considering that the unique MPMA behavior originates from the combination of a first-order hysteretic structural transition between martensitic and austenite phase with a ferromagnetic behavior of the austenite phase. Comparison of such an approach with experimental magnetic property measurements of a Heusler Nickel-CobaltManganese-Indium alloy shows a good ability to predict the low-field magnetic response, highlighting the main involved parameters for further developments.
\end{abstract}

Keywords: Shape Memory Alloys, Metamagnetic materials, Heusler Alloys, thermomagnetic energy conversion

Submitted to: J. Phys. D: Appl. Phys. 


\section{Introduction}

With the recent trends in the development of smart materials, new applications coupling several physical domains have been envisioned in terms of actuation, sensing and energy harvesting for instance. Typical smart materials include for example piezoelectric effect as electromechanical materials ([1]), magnetostrictive materials ([2]) and magnetorheological fluids and elastomers ([3]), both coupling mechanics and magnetism, magnetocaloric devices allowing a magnetic control of the thermal behavior ([4]), and Shape Memory Alloys linking thermal and mechanical domains. Specifically, this last class of materials, also abbreviated SMA, has been the subject of numerous works, due to their unique abilities in providing high stress and strain, reasonable response time, and shape memory capabilities that permit only applying stimuli during changing states $([5,6,6,7,8,9])$.

SMAs are typically based on temperature-dependent phase transition between austenite and martensitic structural configurations. Such a link between thermal and mechanical domains can therefore be taken into advantage for their application as actuators. This phase transition is triggered by heating up or cooling down the material, for instance through electrical stimulus using Joule's effect or through mechanical solicitation ([10]); the former being more often considered for the sake of implementation easiness. Then, the hysteresic behavior originating from the firstorder phase transition enables shape memory effect that can be taken into advantage in the target application.

However, similarly to the large majority of smart materials, SMAs only link two physical domains. Recently, the use of particular Heusler alloys ([11, 12, 13]) allowed introducing a third physical domain, namely the magnetic aspect, in the SMA coupling, enabling the concept of "MultiPhysic Memory Alloys" (MPMA) as a particular multifunctional material. With the inclusion of this third physical field, new applications can be truly envisioned, such as efficient magnetocaloric devices $([14,15,16$, 17, 18]) or thermal energy harvesters $([19,20])$. In particular, tailoring the material to obtain several types of phase transitions such as structural and magnetic ones around the same temperature, and preferably close to room temperature, has been successfully achieved by introducing Indium in Nickel-CobaltManganese compounds for instance $([14,21,22$, 23, 24]). Such materials have shown the remarkable properties of being non-magnetic at low and high temperature $(\leq$ $80{ }^{\circ} \mathrm{C}$ and $\geq 120{ }^{\circ} \mathrm{C}$ ), while exhibiting magnetic activity in between, enabling applications as actuators such as windowed thermal switch or magnetocaloric device ([21]), sensors like temperature window detector and thermal energy harvesting from temperature gradients $([22])$. More specifically, in the context of thermal energy harvesting using heat engines as alternatives to thermoelectric modules, MPMAs feature strong interest, as their magnetic properties arising at relatively high temperature permits placing magnets on cold side, which therefore prevents their demagnetization and considerably simplifies the system design (Figure 1). 


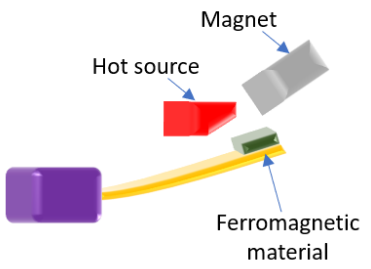
material

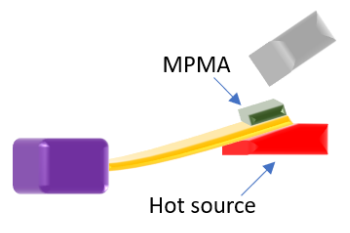

Hot source

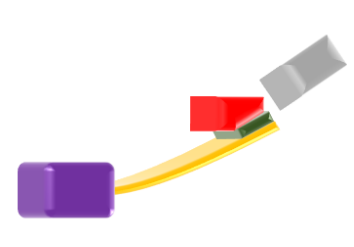

(a) Ferromagnetic

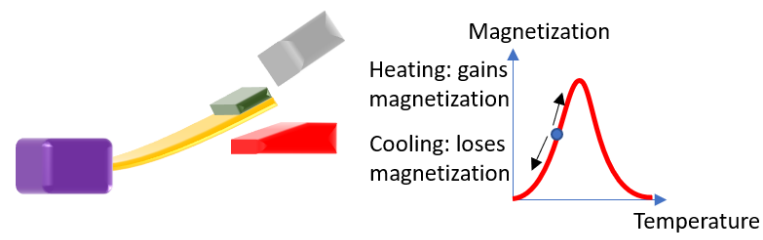

(b) MPMA

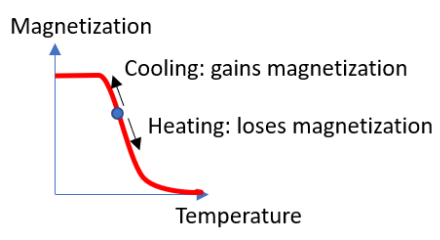

Figure 1. Heat engine using (a) conventional ferromagnetic material (e.g., Gadolinium) and (b) MPMA.

However, the understanding and associated modeling of such MultiPhysic Memory Alloys is still an open question, whose answer would help in giving insights in new material development and/or tailoring for a specific application, including its surrounding environment as well, such as temperature range and typical profile for instance. Hence, the purpose of this paper is to provide a qualitative and quantitative analysis of the mechanisms behind MPMA magnetic behavior. More specifically, the origin of these unique characteristics are considered to arise from the combined structural martensitic-austenite phase transition common to all SMAs along with the ferromagnetic behavior of austenite phase in similar temperature range; the martensitic phase being considered as paramagnetic or non-magnetic in the considered alloy class $([25])$. The paper is organized as follows. Section 2 exposes the elaboration of the material under consideration, consisting in a Heusler alloy based on Nickel-Cobalt-Manganese-Indium compound, along with its lowfield magnetic characterization. These results are then thoroughly interpreted and modeled in Section 3, which ultimately provides a model with physical meaning of the observed magnetic response of the considered material, allowing insights in terms of tailoring and future developments as discussed in Section 4 . Finally, Section 5 briefly concludes the paper by recalling the main findings of the present study. 


\section{Material preparation \& characterization}

\subsection{Synthesis and elaboration}

The material under investigation consists in a Nickel-Cobalt-Manganese-Indium compound whose composition is $\mathrm{Ni}_{45} \mathrm{Co}_{5} \mathrm{Mn}_{36.6} \mathrm{In}_{13.4}$. The interest in such a composition lies in the phase inversion in the temperature diagram, i.e., showing austenite configuration at high temperature and martensitic phase, that is almost paramagnetic, at low temperature thanks to the use of Indium $([26,27])$ compared to other ferromagnetic compounds such as Nickel-Manganese-Gallium alloys ([19]) that feature austenite configuration at low temperature. This yields particular properties in terms of magnetic response as it will be devised latter.

First, as deposited MPMA film was obtained through dual magnetron sputtering of $\mathrm{Ni}_{45} \mathrm{Mn}_{40} \mathrm{In}_{15}$ using Radio Frequency (RF) source at $200 \mathrm{~W}$ and Cobalt from direct current (DC) source with $8 \mathrm{~W}$ power on PolyVinyl Alcohol (PVA) substrate as shown in Figure 2(a). Then the film was peeled of the PVA substrate, resulting in a $5 \mu \mathrm{m}$ film. Finally, a two-step heat treatment was applied in order to obtained modulated martensitic phase at room temperature through crystallization process (Figure 2(b)), leading to the sample shown in Figure 2(c)

\subsection{Magnetic property measurements}

Prior to magnetic characterization, assessment of the structural integrity of the obtained film has been performed through optical measurements and inspection. SEM image of the film after annealing process is depicted in Figure 3(a), showing quite good surface aspect in spite of small grains in localized areas. Further

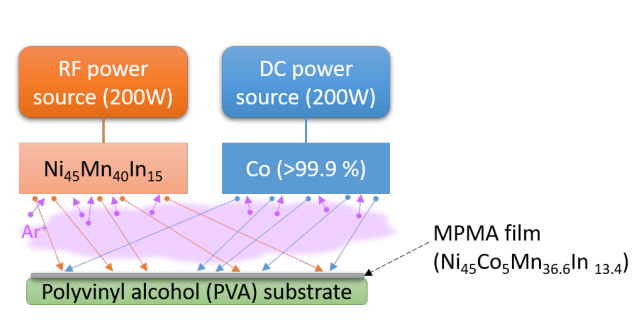

(a) Material elaboration

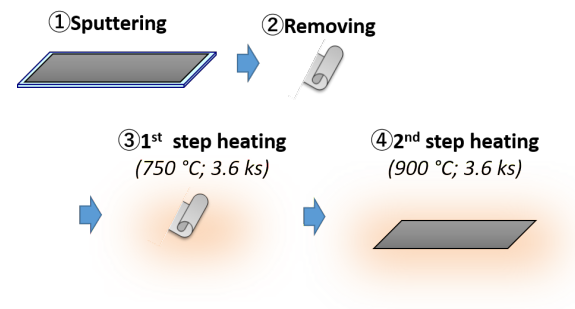

(b) Film fabrication

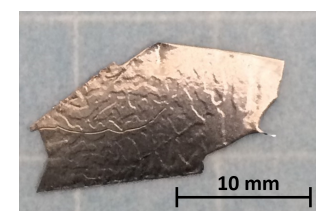

(c) Obtained film

Figure 2. MPMA elaboration steps and film photograph: (a) film synthesis; (b) free-standing film achievement and annealing process (c) Photograph of obtained sample after 2 step annealing process. 


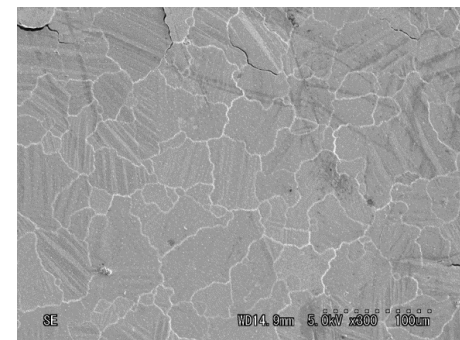

(a) Annealed film SEM image (b)

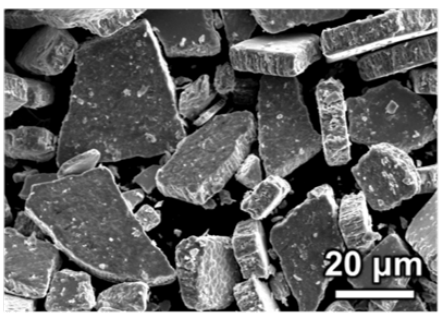

(b) As deposited crushed powder SEM image

Figure 3. SEM imaging of MPMA film.

inspection consisted in crushing the as deposited sample in order to assess regularity along the thickness. Associated SEM image, shown in Figure 3(b), indicates a quite uniform thickness with homogenous powder repartition.

Magnetic measurements were performed using SQUID magnetometer with a MPMA temperature varying from 60 to $125^{\circ} \mathrm{C}$, while the external magnetic flux density was kept constant and equal to $B_{\text {ext }}=50 \mathrm{mT}$. Obtained results (Figure 4), giving the MPMA sample magnetization $M$ under the considered external flux density, were then converted to magnetic susceptibility $\chi$ to assess the intrinsic parameter of the material as:

$$
\chi=\mu_{0} \frac{M}{B_{\text {ext }}}
$$

Under constant magnetic flux density $\left(B_{\text {ext }}\right)$ and in low-field excitation that yields linear response, the susceptibility is directly proportional to the magnetization. Therefore, susceptibility values have also been reported in Figure 4, using a secondary axis (right axis).

Such results demonstrate the ability of the considered MPMA for providing unique magnetic characteristics with a significant magnetic response only in the range of $90-115{ }^{\circ} \mathrm{C}$, confirming the non-magnetic behavior of the martensitic

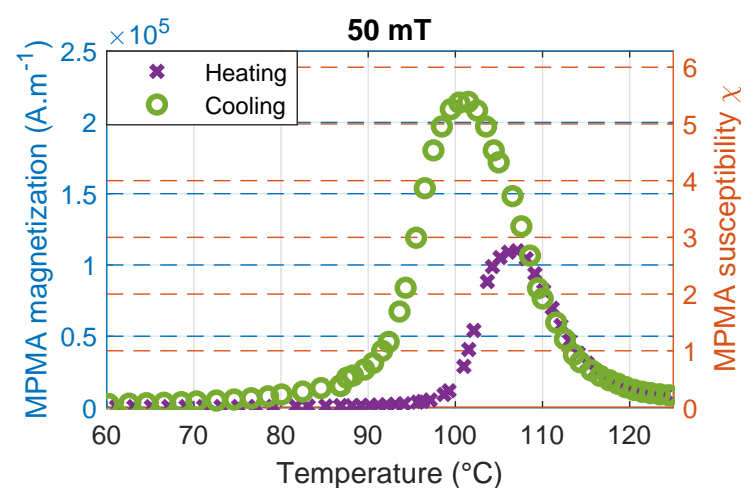

Figure 4. MPMA magnetization (left y-axis) and associated susceptibility (right $\mathrm{y}$-axis) as a function of the temperature for a $50 \mathrm{mT}$ field. 
phase at room temperature. Austenite transition start and stop temperatures have been estimated to respectively 99 and $107{ }^{\circ} \mathrm{C}$ while martensitic ones to 100 and 92 ${ }^{\circ} \mathrm{C}$, respectively. This therefore yields a temperature hysteresis width, defined as the difference between the mean transition temperature of austenite phase $\left(103^{\circ} \mathrm{C}\right)$ and martensitic phase $\left(96^{\circ} \mathrm{C}\right)$, of $7{ }^{\circ} \mathrm{C}$, which is quite adapted for many applications such as actuation or energy harvesting using heat engines. Furthermore, in terms of applications, the negligible magnetization at low temperature provides a significant advantage compared to classical ferromagnetic materials (e.g., Gadolinium - [28, 29]) or ferromagnetic SMAs ([19]) when it comes to device design, as the attracting magnet can be placed on the cold side hence not compromising the magnetization of the latter.

\section{Physical interpretation and modeling}

The objective of this section is to provide physical interpretations of the temperature dependence of the magnetic properties, and more precisely the magnetic susceptibility or permeability. The basic idea behind the proposed model lies in the consideration of two mechanisms: a first-order structural martensitic/austenite phase transition corresponding to the shape memory behavior and a second-order ferromagnetic to paramagnetic phase transition in the magnetic domain for the austenite phase; the martensitic phase being paramagnetic or almost non-magnetic in the considered alloy $([25])$.

\subsection{Structural transition}

The structural transition, from martensitic at low temperatures to austenite at high temperatures and conversely, yields a hysteretic behavior due to latent heat. In the following, it will be considered that the temperature variation is slow so that the system is always at the thermodynamic equilibrium and undergoes quasi-steadystate regime, and that the heating and cooling transitions are symmetric so that the temperature difference to go from one phase to another is the same whatever the considered direction. Furthermore, the transition temperatures are also considered constant whatever the starting point, and only the transition amplitude is affected by this starting point.

The structural phase transition can be obtained through the consideration of the austenite phase ratio $\delta_{A}$. Note that the system being assumed purely biphasic, the martensitic phase ratio $\delta_{M}$ can also be deduced by $\delta_{M}=1-\delta_{A}$. The austenite ratio $\delta_{A}$ can be mathematically derived using two different nondimensional, monotonically increasing functions of the temperature $T([30,31])$. These functions, noted $f_{\text {hyst }}{ }^{+}(T)$ and $f_{\text {hyst }}{ }^{-}(T)$, are defined for all $T \in \mathbb{R}$ with values exactly covering the range $[0,1]$, and correspond to positive and negative 
temperature variations respectively, leading to:

$$
\begin{cases}\mathrm{d} \delta_{A}=\Delta^{+}\left[\frac{\mathrm{d} f_{\text {hyst }}{ }^{+}}{\mathrm{d} T}(T)\right] \mathrm{d} T & \text { for } \mathrm{d} T>0 \\ \mathrm{~d} \delta_{A}=\Delta^{-}\left[\frac{\mathrm{d} f_{\text {hyst }}{ }^{-}}{\mathrm{d} T}(T)\right] \mathrm{d} T & \text { for } \mathrm{d} T<0 \\ \mathrm{~d} \delta_{A}=0 & \text { for } \mathrm{d} T=0\end{cases}
$$

$\Delta^{+}$and $\Delta^{-}$refer to the magnitude of the reverse transformation at the current point, and can be re-evaluated concomitantly with the phase ratio derivative as:

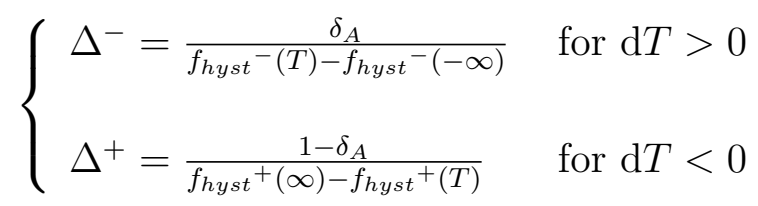

Note that the positive (resp. negative) temperature variation phase transition magnitude is only re-evaluated during negative (resp. positive) temperature slope. It can also be pointed out that such pre-factors can be only evaluated at cancellation or inversion of the temperature derivative for computational effort savings. We also recall that the function shapes are assumed to remain the same whatever the operating point.

In the followings, shifted and weighted sigmoid-like functions, well adapted to SMAs ([32, 33]), are considered as:

$$
\left\{\begin{array}{l}
f_{\text {hyst }}+(T)=\frac{1}{2}\left[1+\tanh \left(\frac{\left.T-\frac{\theta_{A}+\theta_{M}}{2}-\frac{\theta_{A}-\theta_{M}}{2}\right)}{\Theta}\right)\right] \\
f_{\text {hyst }}-(T)=\frac{1}{2}\left[1+\tanh \left(\frac{\left.\left.T-\frac{\theta_{A}+\theta_{M}}{2}+\frac{\theta_{A}-\theta_{M}}{2}\right)\right]}{\Theta}\right)\right]
\end{array}\right.
$$

with $\theta_{A}$ the austenite transition center temperature, defined as the mean of temperatures corresponding to the start and the end of the transition, $\theta_{M}$ the martensitic transition center temperature that follows a similar definition and $\Theta$ the inverse transition slope. Note that the two forward and backward transitions being considered as symmetric, the transition starting and ending temperatures do not explicitly intervene; the symmetry yielding a 3 degree of freedom system instead of 4. It can be drawn from the expression of Eq. (4) that the average of the two transition center temperatures, $\theta_{A+M}=\left(\theta_{A}+\theta_{M}\right) / 2$, relates the center of hysteresis behavior, while their half difference, $\theta_{A-M}=\left(\theta_{A}-\theta_{M}\right) / 2$, denotes the hysteresis width. An alternative writing of this expression, more compact but with a slightly different physical interpretation, consists in solely using the mean austenite and martensitic transition center temperatures as:

$$
\left\{\begin{array}{l}
f_{\text {hyst }}{ }^{+}(T)=\frac{1}{2}\left[1+\tanh \left(\frac{T-\theta_{A}}{\Theta}\right)\right] \\
f_{\text {hyst }}{ }^{-}(T)=\frac{1}{2}\left[1+\tanh \left(\frac{T-\theta_{M}}{\Theta}\right)\right]
\end{array}\right.
$$


as during heating, which corresponds to $f_{\text {hyst }}{ }^{+}(T)$, the transformation goes from martensitic phase to austenite one, and from austenite to martensitic when cooling, where $f_{\text {hyst }}{ }^{-}(T)$ intervenes. Hence, Eq. (5) shows that only the final phase can be considered in the transition, namely austenite for heating and martensitic for cooling. Again, such considerations should be balanced with the assumptions regarding the symmetry of the hysteretic behavior.

Previous experiments reported in Section 2.2 allowed deriving the martensitic and austenite transition center temperatures as $96{ }^{\circ} \mathrm{C}$ and $103{ }^{\circ} \mathrm{C}$, respectively, while the inverse slope transition has been obtained as half the transition starting and ending temperature intervals, given as $107-99=8{ }^{\circ} \mathrm{C}$ for austenite and $100-92=8{ }^{\circ} \mathrm{C}$ for martensitic phases, which confirms the symmetrical effect. This yields the parameters listed in Table 1, leading to the numerical results for the austenite phase ratio depicted in Figure 5. Hence, the proposed model shows abilities in assessing the hysteretic aspect of the phase transformation, including minor loops. Because of considered assumptions and more specifically the symmetric behavior and modification of the sole magnitude, phase ratios at starting austenite transition and at ending martensitic transition are the same in the major loop. The same observation applies to ending austenite transition and starting martensitic transition as well. Finally, it can be noted that due to the smooth transition function as well as experimental uncertainties, these phase ratios at starting and ending transformations are not exactly 0 or 1 .

\subsection{Ferromagnetic transition}

As a unique characteristic of MPMAs, a second phase transition, in the magnetic physical domain, occurs almost simultaneously to the structural phase transition. This phase transition originates from the ferromagnetic nature of the austenite phase yielding a second-order transition, while the martensitic phase is considered as almost paramagnetic. Hence, the expression of the magnetic susceptibility of the austenite phase, noted $\chi_{A}$, is given as:

$$
\chi_{A}=X_{A} \times f_{\text {ferro }}\left(T-T_{\text {ferro }}\right)
$$

where $X_{A}$ is the maximal, low temperature susceptiblity of the austenite phase and $T_{\text {ferro }}$ the ferromagnetic transition temperature. $f_{\text {ferro }}(T)$ is a non-dimensional,

Table 1. Parameters for the structural first-order phase transition.

\begin{tabular}{lr}
\hline Parameter & Value \\
\hline Martensitic transition center temperature $\theta_{M}$ & $96{ }^{\circ} \mathrm{C}$ \\
Austenite transition center temperature $\theta_{A}$ & $103{ }^{\circ} \mathrm{C}$ \\
Inverse transition slope $\Theta$ & $4{ }^{\circ} \mathrm{C}$ \\
\hline
\end{tabular}



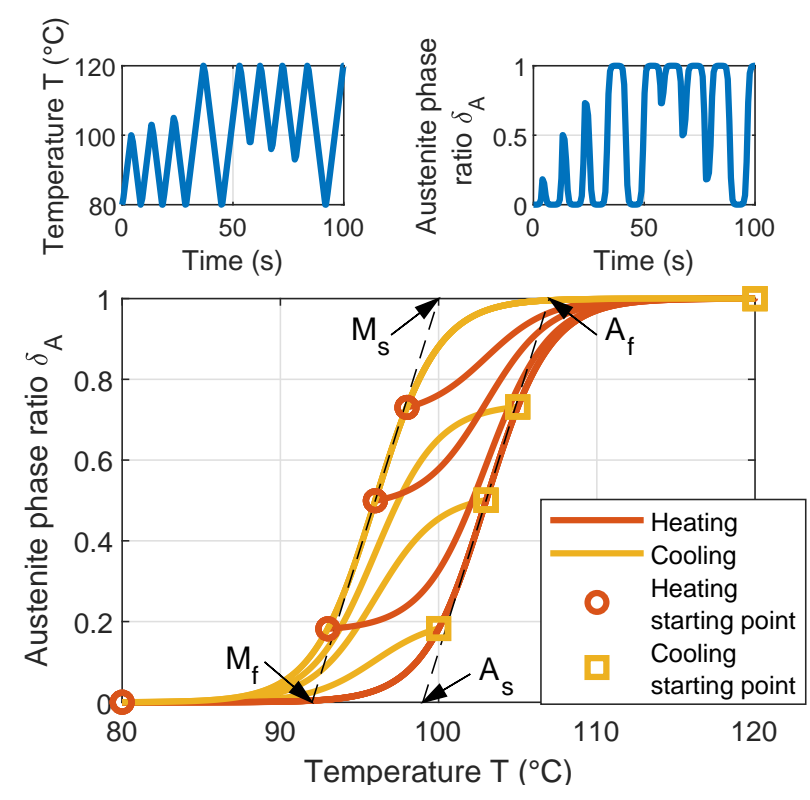

Figure 5. Simulated austenite phase ratio for varying temperature. Top figures show the time-domain applied temperature (left) and associated computed phase ratio (right). Bottom figure depicts the phase ratio dependence to the temperature. $A_{s}$ and $A_{f}$ denote the austenite starting and ending phase transition temperatures, respectively. $M_{s}$ and $M_{f}$ denote the martensitic starting and ending phase transition temperatures, respectively. Starting and ending transition temperature have been obtained through the curves tangent to each branch of the main hysteresis loop at half phase ratio. Red curves represent heating process from points shown as red circles. Orange curves represent cooling process from points shown as orange squares.

monotically decreasing function with low temperature saturation value of 1 , and vanishing towards 0 with increasing temperature. In the following, a hyperbolic saturation function is considered as:

$$
f_{\text {ferro }}\left(T-T_{\text {ferro }}\right)=\frac{1}{2}\left[1-\tanh \left(\frac{T-T_{\text {ferro }}}{\tau}\right)\right]
$$

where $\tau$ denotes the temperature bandwidth coefficient. Using such a function, $T_{\text {ferro }}$ can also be seen as the half-transition temperature where the susceptibility equals $X_{A} / 2$.

Using the parameters listed in Table 2 obtained by fitting the response in full austenite phase area that starts at $107{ }^{\circ} \mathrm{C}$ for heating and ends up at $100{ }^{\circ} \mathrm{C}$ during cooling process, the associated temperature-dependent susceptibility is numerically computed yielding results given in Figure 6. Hence, it can be seen that the ferromagnetic transition actually occurs in a temperature range similar to the structural one (Figure 4). Furthermore, when comparing to experimental results in the full austenite phase region, the choice of the hyperbolic tangent saturation function, along with the considered parameter set, provides a good agreement between theoretical results and experimental measurements. 
Table 2. Parameters for the ferromagnetic transition.

\begin{tabular}{lr} 
Parameter & Value \\
\hline $\begin{array}{l}\text { Initial susceptibility of austenite } \\
\text { phase at low temperature } X_{A}\end{array}$ & 13 \\
& \\
Half-transition $\quad$ temperature & $102{ }^{\circ} \mathrm{C}$ \\
$T_{\text {ferro }}$ &
\end{tabular}

Temperature bandwidth coeffi- $3.5{ }^{\circ} \mathrm{C}$ cient $\tau$

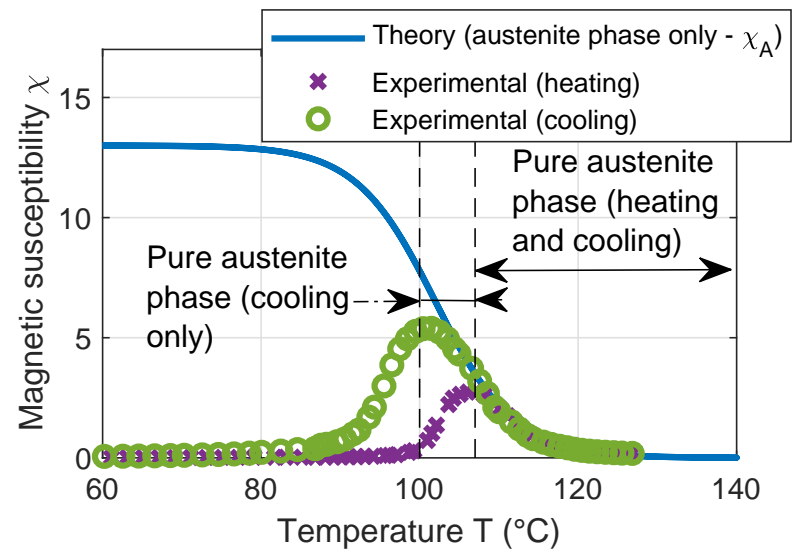

Figure 6. Simulated magnetic response of austenite phase and comparison with measurements.

\subsection{Global response}

The previous two sections allowed the derivation of the austenite phase ratio $\delta_{A}$, the martensitic one $\delta_{M}=1-\delta_{A}$ as the system is considered as purely biphasic and the magnetic susceptibility of the sole austenite phase. However, since the two phases may coexist during the structural phase transition, the global MPMA relative permeability $\mu$ can be obtained considering martensitic and austenite phase permeabilities, respectively referred as $\mu_{M}$ and $\mu_{A}$, through a mixing rule. In the following, a Maxwell-Garnett mixing rule is considered, yielding:

$$
\mu=\frac{\left(1+2 \delta_{A}\right) \mu_{A}+2\left(1-\delta_{A}\right) \mu_{M}}{\left(1-\delta_{A}\right) \mu_{A}+\left(2+\delta_{A}\right) \mu_{M}} \mu_{M}
$$

where the austenite phase relative permeability $\mu_{A}$ is linked to its susceptibility $\chi_{A}$ through:

$$
\mu_{A}=1+\chi_{A}
$$

It can be noted that while Maxwell-Garnett mixing rule is usually used for low volume fraction in dielectric media, it will be considered here valid for the whole 
range of phase ratio. Finally, the magnetic susceptibility $\chi$ of the MPMA can be obtained from its relative permeability $\mu$ as:

$$
\chi=\mu-1
$$

Considering that the martensitic phase shows paramagnetic behavior with a constant relative permeability $\mu_{M}=1.1$ and using parameters from Tables 1 and 2 yield the results depicted in Figure 7. As expected, the low temperature permeability is equal to the martensitic one that has very low value. As the temperature increases, the structural phase transition is initiated and the permeability starts increasing as the austenite phase ratio increases. Further increasing the temperature however leads to a decrease of the permeability, as the ferromagnetic behavior of the austenite phase leads to a decrease of the associated magnetic activity. From high to low temperature variations, the permeability first experiences an increase as the magnetic activity of the austenite phase becomes more important, but then decreases due to the structural transition to martensitic phase that exhibits low magnetic permeability. Also, it could be noted that the maximal permeability during cooling phase is higher than when heating, as the hysteretic behavior in the structural phase transition yields a longer lasting presence of the austenite phase. Comparison with values extracted from the experimental analysis are also depicted in Figure 7, which shows very good agreement with theoretical predictions. It can however be noted some discrepancies in the low temperature range of cooling stage, probably due to an asymmetry in the structural phase transition, with the austenite to martensitic phase transition being different from the converse one.

Figure 8 shows the expectable relative permeability of the MPMA considering minor cycles in temperature. Although no associated experiment was performed, the considered model for the first-order transition is quite well-established ([32, 33]) to consider the curves depicted in Figure 8 to be reasonably close to the actual material response. While the global envelope of the permeability profile is not modified in

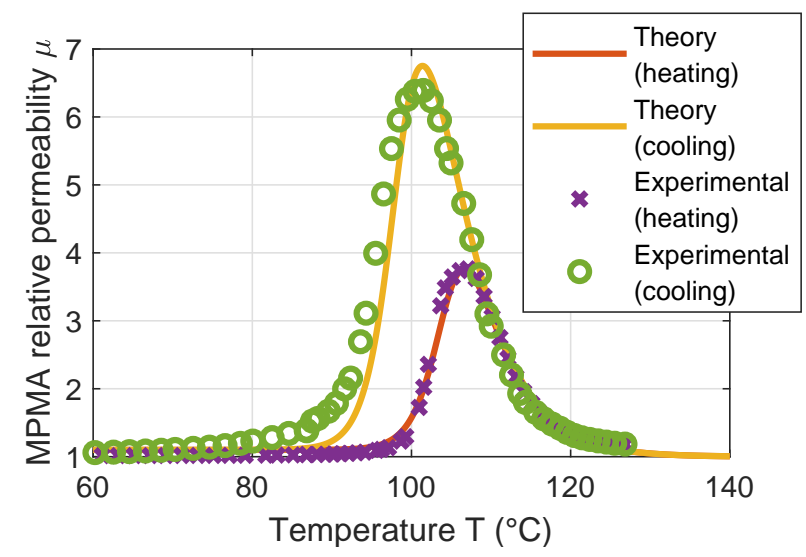

Figure 7. Calculated MPMA relative permeability vs. experimental measurements. 


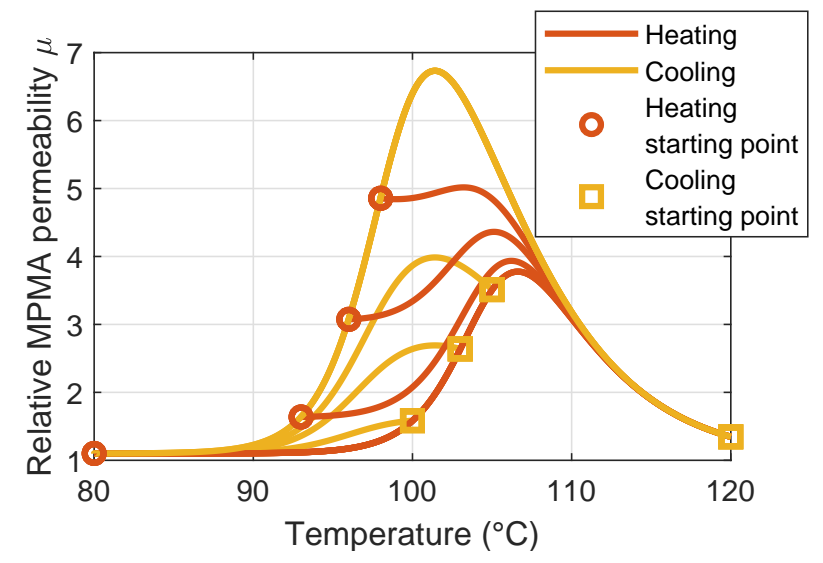

Figure 8. Calculated MPMA relative permeability considering minor cycles. Red curves represent heating process from points shown as red circles. Orange curves represent cooling process from points shown as orange squares.

minor loops due to the assumption that the hysteretic function shape is not affected except for its magnitude, decreasing the temperature before the full austenite phase transformation yields a plateau or even an increase for large starting austenite phase ratio, without however reaching the maximal permeability obtained with a full cycle. The same observation can be drawn when the temperature is increased before full martensitic transformation. It can be noted that the permeability peak positions are also changing when performing minor loops, as they are moving towards low temperatures when decreasing the temperature before full austenite transformation and towards high temperatures when increasing the temperature before full martensite transformation. Such changes in the optimal permeability temperature can be of significant importance when the MPMA is operating in relatively limited temperature ranges.

\section{Discussion \& tailoring possibilities}

Based on the previous model, this section aims at discussing the effect of considered parameters on the MPMA response, in the framework of tailoring/optimizing the material for a specific target application. In that sense, a summary of used parameters, either obtained through experimental analysis for structural properties as per Table 1 or by fitting process for magnetic properties as explained in Section 3.2, are given in Table 3, providing a basis for further considerations. Note that the magnetic response is related to the magnetic susceptibility and permeability, and not directly to magnetization under low external field. This is explained by the fact that the magnetization is not only strongly dependent on external conditions, for instance the magnetic flux density, but also on the structural transition properties of the MPMA. For example, a lower transition temperature would yield higher apparent susceptibility and thus magnetization, with these properties being possibly made 
Table 3. Main model parameters.

\begin{tabular}{|c|c|c|}
\hline Parameter & $\begin{array}{l}\text { Variable } \\
\text { (condition if } \\
\text { applicable) }\end{array}$ & $\begin{array}{l}\text { Initial } \\
\text { value }\end{array}$ \\
\hline \multicolumn{3}{|l|}{ Structural properties } \\
\hline Martensitic transition center temperature & $\theta_{M}$ & $96{ }^{\circ} \mathrm{C}$ \\
\hline Austenite transition center temperature & $\theta_{A}\left(>\theta_{M}\right)$ & $103^{\circ} \mathrm{C}$ \\
\hline Inverse structural transition slope & $\Theta$ & $4^{\circ} \mathrm{C}$ \\
\hline \multicolumn{3}{|l|}{ Magnetic properties } \\
\hline Half-ferromagnetic transition temperature & $T_{\text {ferro }}$ & $102^{\circ} \mathrm{C}$ \\
\hline Ferromagnetic temperature bandwidth coefficient & $\theta$ & $3.5^{\circ} \mathrm{C}$ \\
\hline $\begin{array}{l}\text { Low temperature susceptibility of austenite phase } \\
\text { (ferromagnetic phase) }\end{array}$ & $X_{A}$ & 13 \\
\hline $\begin{array}{l}\text { Relative permeability of martensitic phase (paramag- } \\
\text { netic phase) }\end{array}$ & $\mu_{M}$ & 1.1 \\
\hline
\end{tabular}

varying in the following analysis.

\subsection{Effect of austenite phase permeability}

Because of the combined effect of structural and ferromagnetic transitions, the change of the austenite phase susceptibility does not yield a simple increase or decrease of the global permeability. Instead, the full response is changed. Figure 9(a) depicts the effect of such a change, keeping constant all the other parameters. Hence, it can be observed that the temperature corresponding to the maximum permeability is indeed varying and increases with the initial susceptibility, but in a rather limited way as results indicate variations from $105.12{ }^{\circ} \mathrm{C}$ to $108.16{ }^{\circ} \mathrm{C}$ when heating and from $99.84{ }^{\circ} \mathrm{C}$ to $103.04{ }^{\circ} \mathrm{C}$ when cooling. A slightly more pronounced effect in heating stage can be found however. Another interesting aspect of this analysis is that the maximal permeability is increased compared to the initial permeability of the austenite phase, which is caused by the mixed phases, where the martensitic phase features low permeability. However, such an increase is less than the sole austenite phase maximal permeability modification. 

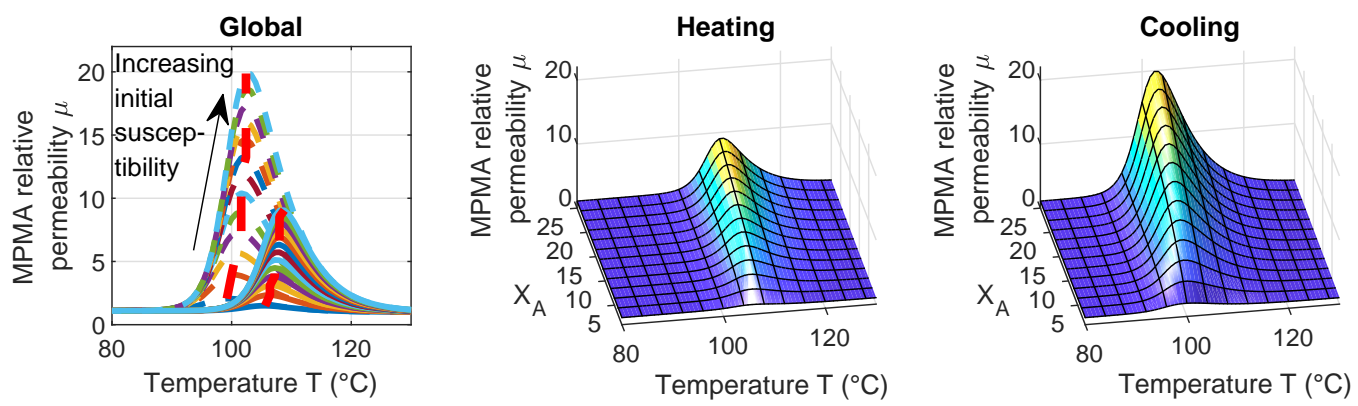

(a) Varying initial susceptibility of the austenite phase $X_{A}$ (from 2 to 25 by increment of 2)
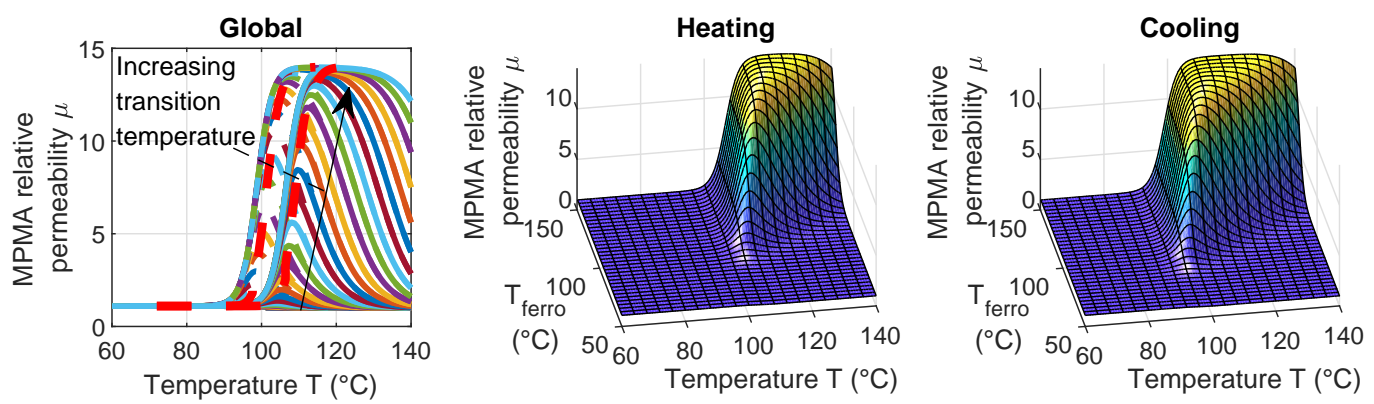

(b) Varying ferromagnetic transition temperature of the austenite phase $T_{\text {ferro }}$ (from $50{ }^{\circ} \mathrm{C}$ to $149{ }^{\circ} \mathrm{C}$ by increment of $3{ }^{\circ} \mathrm{C}$ )
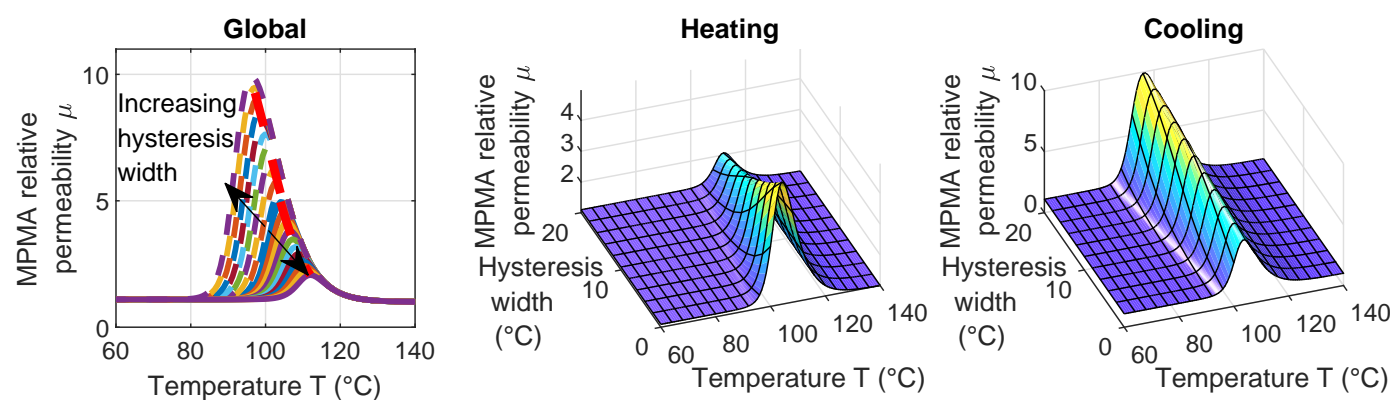

(c) Varying structural transition hysteresis width (from $0{ }^{\circ} \mathrm{C}$ to $20{ }^{\circ} \mathrm{C}$ by increment of $2{ }^{\circ} \mathrm{C}$ )

Figure 9. Calculated MPMA relative permeability under different conditions: (a) varying maximal austenite susceptibility; (b) varying austenite ferromagnetic transition temperature and (c) varying structural transition width. Plain lines refer to heating case and dashed lines to cooling. Thick red lines denote the maximum permeabilities in both heating and cooling stages.

\subsection{Effect of temperature matching}

The other effect of interest is the location of the ferromagnetic transition temperature, namely $T_{\text {ferro }}$, with respect to the average of center structural transition temperatures $\theta_{A+M}=\left(\theta_{A}+\theta_{M}\right) / 2$. Results depicted in Figure 9(b) show that increasing the ferromagnetic transition temperature first yields an increase of the maximal permeability along with an increase of the associated temperature, but, after a particular value of this parameter, a plateau appears which is explained by the fact that the austenite phase susceptibility remains constant and equals to 
its initial value during the whole structural transition process. In this case, and because the two transitions no longer occur simultaneously, the hysteretic structural transition is fully reflected by the magnetic properties.

\subsection{Effect of structural transition width}

Finally, the last considered variable parameter under investigation in this section is the difference between the martensitic and austenite center temperatures in the hysteretic structural transition. Varying such a parameter leads to results shown in Figure 9(c), Unlike previous cases, simultaneously increasing the austenite transition temperature and decreasing the martensitic one not to change the center temperature leads to a decrease (resp. increase) of the peak permeability with an increase (resp. decrease) of the associated temperature in heating (resp. cooling) stage. Such an observation is consistent with the ferromagnetic behavior of the austenite phase, which yields a lower (resp. greater) susceptibility when the transition temperature is increased (resp. decreased). Specifically, the increase of the permeability during cooling may be seen as beneficial in the framework of magnetic field actuated systems, but it should be kept in mind that in this case the latter should operate over a larger temperature range.

\subsection{Tailoring in the framework of thermal energy harvesting using heat engines}

In the framework of thermal energy harvesting using heat engines (Figure 1, [19]), this section aims at giving some indications regarding the material selection and tailoring. More precisely, the following characteristics are desirable to this end:

(i) Transition temperature matching (structural and magnetic) in the vicinity of the mean working temperature

(ii) Sharp structural transition

(iii) Reduced hysteresis

(iv) Significant magnetic response of the austenite phase

4.4.1. Close transition temperatures in the vicinity of the mean working temperature: With the application in mind, the transition temperatures should be consistent with the targeted environment. In addition, as shown in Figure 9(b), the ferromagnetic transition temperature of the austenite phase should not be significantly lower than the structural transition temperature, and significantly higher value is actually desirable. Otherwise, the magnetic response would be wiped out by the structural transition, and the device would not be attracted by the magnet. To address these issues, the addition of Cobalt is a way to tune the temperature ([34]), along with Indium ([27, 35, 36]). Although out of the scope of the present study focusing on low-field response, transition temperature may also be slightly changed by the application of a strong external magnetic field ([21]). 
4.4.2. Sharp structural transition: Ensuring high operating frequency and/or allowing operation over a narrow temperature range necessitates quick transition from martensitic to austenite phase and conversely, which is reflected by the inverse transition slope $\Theta$ in the proposed model. In addition of playing with the composition ratio, exploring other components combined with Nickel, Manganese and Cobalt is a possible route. As an example, antimony ([27]) or tin ([37, 38, 39, 40]) can be considered to this end, although the former solution yields larger structural transition hysteresis and the latter higher transition temperatures.

4.4.3. Reduced hysteresis: The hysteresis may be an issue in order to ensure device operations, as it either reduces the operating frequency and thus energy exchange and potential output power, or can simply make the device non operating when the experienced temperature range is limited. Hence, ensuring martensitic to austenite and converse transitions as close as possible to each other is of particular importance. Furthermore, as shown in Figure 9(c), increasing the width of transition pushes the austenite response towards high temperature, hence reducing the magnetic response while heating. Then, the device may not be attracted by the magnet (Figure 1, [22]) preventing the correct operation of the device. Ways of limiting such a hysterestic effect lie in avoiding defects in the alloy as well as playing with the composition ratio of each component $([27])$.

4.4.4. Significant magnetic response: For triggering the thermally-induced vibrations, the MPMA needs to get attracted by the magnet, requiring adequate magnetic properties. In addition of ensuring that the ferromagnetic transition occurs at sufficiently higher temperature than the structural one as discussed in Section 4.4.1, the increase of the austenite low-temperature susceptibility, for instance through the composition ([34, 37]), is also a straightforward way (Figure 9(a)). However, it should be noted that, while the increase of this parameter may have beneficial effect during heating, the even more significant increase of the magnetic response during cooling stage requires going down to lower temperatures for effectively demagnetizing the device, hence possibly compromising the application in the case of limited working temperature range. When possible, changing transition conditions may therefore be preferable. Globally, a change in material morphology, such as thin films or powders, lamination of films, plastic working or heat treatment and so on may also lead to changes in magnetic properties (hysteresis and maximal magnetic response for instance) towards beneficial effects in the framework of the envisioned application.

\section{Conclusion}

In order to better understand the underlying mechanisms in the magnetic response of MultiPhysic Memory Alloys (MPMAs) that combine three different 
physical domains, namely thermal, structural and magnetic, in the framework of energy harvesting device tailoring and optimization, this study proposed a theoretical analysis based on structural and magnetic phase transitions to predict the permeability of the material. The model considers that the material may feature simultaneously a first-order hysteretic structural phase transition, from paramagnetic martensitic phase at low temperature to ferromagnetic austenite phase at high temperature, and a ferromagnetic transition of the austenite phase, exhibiting a decrease in the associated magnetic susceptibility as the temperature increases. Combining these two effects therefore shows a hysteretic behavior of the global MPMA permeability, with a maximum value of the latter occurring both at heating and cooling phases; the maximum permeability value being much larger when temperature is decreased. Comparison of theoretical predictions with experimental measurements conducted on a Nickel-Cobalt-Manganese-Indium compound showed very good agreement. Finally, the study proposed the investigation of the effect of some parameters on this magnetic response, highlighting some insights in terms of material elaboration for optimizing or tailoring the associated devices in accordance to the target application and environment, particularly in the framework of thermal energy harvesting through heat engines.

\section{Acknowledgements}

M. Lallart, L. Yan and H. Miki gratefully acknowledge the support of JSPS through invitational fellowship grant number L19530 and postdoctoral fellowship grant number PE19727. M. Lallart is also grateful to INSA-Lyon for its support through the CRCT program.

\section{ORCID iDs}

Mickaël Lallart https://orcid.org/0000-0002-6907-5946

\section{Conflict of interest}

The authors declare that they have no conflict of interest.

\section{References}

[1] Trolier-McKinstry S, Zhang S, Bell A J and Tan X 2018 Ann. Rev. Mater. Res. 48191.

[2] Elhajjar R, Law C-T and Pegoretti A 2018 Prog. Mater. Sci. 97204

[3] Choi S B and Han Y M 2017 Magnetorheological Fluid Technology: Applications in vehicle systems (Boca Raton, USA: CRC Press, Taylor and Francis) ISBN: 9781138076365

[4] Tishin A M, Spichkin Y I, Zverev V I and Egolf P W 2016 Int. J. Refrig. 68177

[5] Bellouard Y 2008 Mat. Sci. Eng. A-Struct. 481-482 582

[6] Nespoli A, Besseghini S, Pittaccio S, Villa E and Viscuso S 2010 Sens. Actuator A-Phys. 158 149 
[7] Mohd Jani J, Leary M, Subic A and Gibson M A 2014 Mater. Des. 561078

[8] Lobo P S, Almeida J and Guerreiro L 2015 Procedia Engineering 114776

[9] Choudhary N and Kaur D 2016 Sens. Actuator A-Phys. 242162

[10] Ko W-S, Maisel S B, Grabowski B, Bae Jeon J and Neugebauer J 2017 Acta Mater. 12390

[11] Webster P J 1969 Contemp. Phys. 10559

[12] Graf T, Parkin S S P and Felser C 2011 IEEE Trans. Magn. 47373

[13] L'vov V A, Cesari E, Pérez-Landazábal J I, Recarte V and Torrens-Serra J 2016 J. Phys. D: Appl. Phys. 49205004

[14] Planes A, Mañosa L and Acet M 2009 J. Phys.-Condes. Matter 21233201

[15] Caballero-Flores R, Sánchez-Alarcos V, Recarte V, Pérez-Landazábal J I and Gómez-Polo C 2016 J. Phys. D: Appl. Phys. 49205004

[16] Ossmer H, Wendler F, Gueltig M, Lambrecht F, Miyazaki S and Kohl M 2016 Smart Mater. Struct. 25085037

[17] Bruederlin F, Ossmer H, Wendler F, Miyazaki S and Kohl M 2017 J. Phys. D: Appl. Phys. 50424003

[18] Hou H, Simsek E, Stasak D, Al Hasan N, Qian S, Ott R, Cui J and Takeuchi I 2017 J. Phys. D: Appl. Phys. $\mathbf{5 0} 404001$

[19] Gueltig M, Wendler F, Ossmer H, Ohtsuka M, Miki H, Takagi T and Kohl M 2017 Adv. Energy Mater. 71601879

[20] Farsangi M A A and Zohoor H 2019 J. Phys. D: Appl. Phys. 52135501

[21] Bourgault D, Tillier J, Courtois P, Maillard D and Chaud X 2010 Appl. Phys. Lett. 96132501

[22] Gueltig M, Ossmer H, Ohtsuka M, Miki H, Tsuchiya K, Takagi T and Kohl M 2014 Adv. Energy Mat. 41400751

[23] Danilevich A G and L'vov V A 2016 J. Phys. D: Appl. Phys. 49105001

[24] Chabri T, Ghosh A, Nair S, Awasthi A M, Venimadhav A and Nath T K 2018 J. Phys. D: Appl. Phys. 51195001

[25] Bruno N M, Huang Y J, Dennis C L, Li J G, Shull R D, Ross J H Jr., Chumlyakov Y I and Karaman I 2016 J. Appl. Phys. 120075101

[26] Kohl M, Gueltig M, Pinneker V, Yin R, Wendler F and Krevet B 2014 Micromachines 5 1135

[27] Sutou Y, Imano Y, Koeda N, Omori T, Kainuma R, Ishida K, Oikawa K 2004 Appl. Phys. Lett. 854358

[28] Fraga G L F, Pureur P and Cardoso L P 2010 J. Appl. Phys. 107053909

[29] Ujihara M, Carman G P and Lee D G 2007 Appl. Phys. Lett. 91093508

[30] Likhachev A A 1995 Scripta Metall. Mater. 32633

[31] Shishkovsky I 2012 Physics Procedia 39893

[32] He J and Toi Y 2013 Journal of Solid Mechanics and Materials Engineering 711

[33] Zhang J, Yin Y and Zhu J 2013 Sens. Actuator A-Phys. 201264

[34] Kainuma R, Imano Y, Ito W, Sutou Y, Morito H, Okamoto S, Kitakami O, Oikawa K, Fujita A, Kanomata T, Ishida K 2006 Nature 439957

[35] Khovaylo V V, Kanomata T, Tanaka T, Nakashima M, Amako Y, Kainuma R, Umetsu R Y, Morito H and Miki H 2009 Phys. Rev. B 80144409

[36] Buchelnikov V D, Sokolovskiy V V, Taskaev S V, Khovaylo V V, Aliev A A, Khanov L N, Batdalov A B, Entel P, Miki H and Takagi T 2011 J. Phys. D: Appl. Phys. 446

[37] Srivastava V, Song Y, Bhatti K and James R D 2011 Adv. Energy Mater. 197

[38] Khovaylo V, Koledov V, Shavrov V, Ohtsuka M, Miki H, Takagi T and Novosa V 2008 Mat. Sci. Eng. A-Struct 481-482 322

[39] Khovaylo V V, Skokov K P, Gutfleisch O, Miki H, Takagi T, Kanomata T, Koledov V V, Shavrov V G, Wang G, Palacios E, Bartolomé J and Burriel R 2010 Phys. Rev. B 81 214406

[40] Khovaylo V V, Skokov K P, Gutfleisch O, Miki H, Kainuma R and Kanomata T 2010 Appl. 
Phys. Lett. 97052503 